\title{
The Construct of Change in Close Relationships
}

\author{
Anna H. C. Neo', Weining C. Chang² \\ ${ }^{1}$ Nanyang Technological University, Singapore City, Singapore \\ ${ }^{2}$ Institute of Mental Health, Singapore City, Singapore \\ Email:neo.nnchin@yahoo.com
}

How to cite this paper: Neo, A. H. C., \& Chang, W. N. C. (2017). The Construct of Change in Close Relationships. Psychology, $8,1188-1215$

https://doi.org/10.4236/psych.2017.88077

Received: April 11, 2017

Accepted: June 20, 2017

Published: June 23, 2017

Copyright (C) 2017 by authors and Scientific Research Publishing Inc. This work is licensed under the Creative Commons Attribution International License (CC BY 4.0).

http://creativecommons.org/licenses/by/4.0/ c) (i) Open Access

\begin{abstract}
Relationship beliefs are individual's cognitive representations of interpersonal relationships found to contribute to the functioning, development and resolution of close relationships. The present study extends Rogge and Bradbury's (2002) multidimensional approach to the understanding of relationship change by exploring the construct of change that involves both intentional interventions and naturally occurring changes. We conducted two studies on Singaporean Chinese adults, aged 18 to 29 years old. Study 1 explored the laypeople's construct of relationship change through qualitative interviews. The responses were utilized to develop the Relationship Beliefs about Change (RBC) scale. Study 2 aimed to validate the RBC scale and found three dimensions: Agent of Change (AGC), Inevitable Change (IC), and Managing Change (MC). The RBC demonstrated adequate psychometric properties as an assessment of change beliefs in relationship. The scale revealed a multifaceted system of beliefs consisting of the constantly changing nature of relationship: AGC-who or what changes; IC-the belief system about change, and $\mathrm{MC}-$ the need for intervention to curb changes in relationships.
\end{abstract}

\section{Keywords}

Change, Relationship Beliefs, Implicit Beliefs, Close Relationships, Romantic Relationships, Scale Validation

\section{Introduction}

\subsection{The Construct of Change in Close Relationships}

It is essential for people to hold theories and beliefs about themselves and others to regulate their social interactions (Fletcher, et al., 2006). Recent studies in intimate relationships have explored the change-related beliefs people bring to their relationships. However, discussions regarding "change" in human attributes and relationships are mainly concerned with malleability, the potential to change or mold. Rogge and Bradbury (2002) proposed a multidimensional ap- 
proach towards the understanding of change in intimate relationships to include both induced change (i.e., managed change) and natural change (i.e., spontaneous or naturally occurring changes overtime).

The original intention for using such terminology was to refer "induced change" versus "natural change" as "experimental change" versus "natural change" observed in non-experimental studies (Rogge \& Bradbury, 2002). These researchers suspected that "natural change" might entail more lasting and ubiquitous effects on relationships, especially marital relationships. The underlying mechanisms involved in induced change and those involved in natural change might be different, thus preventing an accurate and comprehensive understanding of relationship change.

Building on this perspective, the current study aimed to expand the previous theoretical notion of contrasting induced changes against naturally occurring changes. For the present study, the construct of "malleability" refers to the potentiality of a relationship to be changed by deliberate effort. It is conceptualized in such way that relationship malleability could involve induced changes in the relationship as a result of purposive action by the self or the partner in the relationship, whilst natural change is a change that occurs without any deliberate intervention or purposeful effort. Natural change is seen as the natural course of the relationship or the unfolding of the relationship's inherent developmental process.

Although past researchers did not define change in relationships to explicitly exclude changes that are naturally occurring, the research focus has primarily been on induced changes. As found in research in human intelligence, incremental theorists of intelligence attributed negative outcomes of achievement tasks to inadequate effort applied (i.e., lacking of deliberate action) rather than naturally occurring events or natural endowments (Dweck, Chiu, \& Hong, 1995). In the relationship context, people who subscribed to the growth theory of relationship (Knee \& Petty, 2013) were less concerned about problems in their relationships, as they believed that they were able to do something about the problem, thereby leading to further development or growth of the relationship (Knee, 1998). Similarly, dysfunctional beliefs measured by Relationship Belief Inventory (Epstein \& Eidelson, 1982), such as attributing to "partner cannot change", emphasized the ability or motivation of the partner to introduce change (James, Hunsley, \& Hemsworth, 2002). For instance, "if my partner wants to change, I believe he/she can do it", and "a partner can learn to become more responsive to his/her partner's needs" (Epstein \& Eidelson, 1982). Therefore, it is apparent that these studies focused on the partners' ability or motivation to change as the concerned attributes, instead of the characteristics of the relationship itself.

Naturally occurring changes of relationships were either overlooked or not given equal attention in relationship research. An adage such as "time changes everything" often carries a negative connotation especially for romantic relationships. In the relationship context, partners are more often concerned about their relationship's potential deterioration as a result of mere lapse of time (Hinde, 1997; Rogge \& Bradbury, 2002). Moreover, beliefs about natural changes are cognitions about the dyad, rather than the individual. For instance, one may 
believe that, given sufficient time, an intimate relationship will change. This clearly indicates the belief concerns the dyad as a whole, rather than the behavior of any specific partner. Apparently, natural change has often been a concern in intimate relationship. It is probable that both induced and natural changes are important to the relationship representations that involve the dyad. Following the above arguments, the first objective of the present study was to conceptualize the construct of changes in close relationships, and further, to develop a comprehensive measurement to assess the beliefs about the changes in intimate relationships.

In sum, relationship change is a multifaceted and complex phenomenon. Beliefs about changes in relationships should be explored by taking into consideration the experiences of and lay beliefs about relationship changes. The conceptualization of change beliefs in relationships would therefore involve both natural changes (process of development as time goes by) and purposively induced changes (active intervention with aim to bring about change). In addition, there might be different beliefs underlying or shaping the perception of changes.

\subsection{Cultural Basis of Relationship Beliefs}

Like most social cognition constructs, relationship beliefs are socially constructed. These beliefs could only be validly understood in the cultural context in which it is constructed: whereby the meaningful system of shared relationship beliefs informs the formation, interpretation and potential dissolution of relationships. Relationship-related representations, as is the case with most knowledge structures, can be derived not only from first-person direct experiences in romantic relationships, but also from indirect sources such as shared relationship beliefs of family and friends, cultural norms and the media (Hatfield \& Rapson, 2010). Culture provides conceptual models of intimate relationships. The same beliefs may produce different results in different cultural contexts.

Indeed, relationships are embedded within the culture and hence, are intimately affected or directed by the culture (Hatfield \& Rapson, 2010). Specifically, Goodwin and Tang (1996) noted that culture governs and guides the love styles people adopt in romantic relationships. The Chinese relationship model (Chan, Ng, \& Hui, 2010), being collectivistic, emphasizes fulfilling the responsibilities and obligations of one's role in the relationship. Furthermore, in the closely-knit Chinese communities, high interdependent, mutually bonding relationships are valued (Tang \& Zuo, 2000, as cited in Chan, Ng, \& Hui, 2010). In this context, love and romance between individual partners are considered less important than the familial responsibilities and obligations that the partners are expected to honor (Moore \& Leung, 2001). Indeed, the great emphasis on familial relationships is evident across not only the Chinese, but also across the varied and diverse collectivistic societies, such as Japan and Korea (Kagitcibasi, 2007). It is likely that the people who adhere more to the traditional collectivistic orientation, such as Chinese, Japanese, and even South American, construct relationship models differently from people who are from the more individualistic societies (Gaines, Buriel, Liu, \& Rios, 1997). However, a large proportion of the re- 
lationship belief studies were conducted in the individualistic context or based on the modern Western ideologies about relationships (e.g., Knee, 1998; James, et al., 2002). Hence, it is unclear whether in the collectivistic Asian context, with its rich cultural traditions, relationship beliefs would differ from those found in the Western cultural context.

Relationship behaviors have been found to differ across the individualist/collectivist divide: for instance, Chinese were found to utilize style that is more obliging rather than confrontational in interpersonal interactions, when compared to their Western counterparts in managing conflicts in romantic relationships (Liu, 2012). These findings about Chinese or Asian styles of cognition and behaviors probably stemmed from the Chinese philosophy of the "way of life," for instance, "Zhong Yong" (i.e., take the middle way or moderation), by being modest, to avoid an inclination towards the extremes (Ji, Lee, \& Guo, 2010). Within the Asian tradition, maintaining harmony in the relationship is the primary concern for the wellbeing of the relationship. In the traditional Chinese worldview, the wellbeing of the collective and the wellbeing of the relationship are considered more important than the wellbeing of the individual partners. This is especially apparent when dealing with conflicts in relationships, in which case, the principle of "Zhong Yong" informs the individual to be moderate towards others' opinions and needs, and readily accommodate to them in resolving conflict (Ji et al., 2010). This typically results in an effort to change oneself rather than attempting to change the people in their social environment (Chang, Chua \& Toh, 1997). This is mainly achieved by regulating and avoiding the expression of extreme emotions for the good of the individual, relationship and society. On the other hand, studies have shown that the Chinese are more likely to adopt anxious attachment styles that motivate them to be more alert and attentive to the relationship status quo (Chan, Ng, \& Hui, 2010). Being anxiously attached might be an adaptive fit to the highly interdependent relationship network in the collective cultures, such as Japan and Korea (Triandis, 1989).

Therefore, the present study aimed to explore the indigenous beliefs about relationships and relationship change. Furthermore, based on the results, we constructed a measure of relationship beliefs about change in the Asian context. Views related to beliefs about change and relationship changes, as well as how these beliefs might influence their relationship behaviors were collected from a sample of young people below 30 years old in the Singaporean Chinese community.

\section{Methods}

\subsection{Study 1: Conceptualization of the Construct of Change}

\subsubsection{Participants}

Fifty-one undergraduate Singapore university students, 26 males and 25 females, were recruited to take part in a one-to-one, semi-structured interview. These students enrolled in an introductory psychology course and obtained partial course credit for their participation. All of them were citizens or permanent res- 
idents of Singapore, of age ranging from 18 to 28 years old with mean age of $22.57(S D=1.19)$ for male participants and $20.17(S D=1.37)$ for female participants. Only students of Chinese ethnicity were recruited. All participants were involved in romantic relationships at the time of the interview.

\subsubsection{Design and Procedures}

The interviews were digitally recorded with the participant's consent and institution approval. Each interview took about 30 to 45 minutes, with the first five minutes dedicated to building rapport between interviewees and the interviewer. The semi-structured interviews consisted of a list of questions developed to test the research question concerning personal beliefs about romantic relationships. Participants were asked about their expectations and beliefs regarding intimate relationships, including their personal beliefs about the nature and causes of potential changes in the relationship. The participants were also interviewed about what they might do when the relationship changes. These questions were derived from literature review in regard to relationship beliefs and adapted to fulfil the aims of the present study.

The main question asked about relationship change was, "Will relationships change and what are the factors that contribute to changes in a relationship?" This question was followed by probing questions such as, "Is change in a relationship something good?", "Do people have control over their relationships or changes in relationships?", "Would you want to make changes to your relationship if you have a choice, and why would or would you not do so?"

Responses from the interviews were then transcribed by two research assistants who were students from psychology honors class in a local university. The researchers, who were authors of the paper, identified the emergent themes from the interview scripts by searching for recurrent concepts that were pertinent to relationship beliefs about change. Subsequently, the research assistants independently coded the recurrent themes by the frequency of the interview responses that converge on the themes. The inter-rater reliability was found to be $98 \%$, computed from percentage agreement (i.e., number of agreement scores divided by total number of scores). The frequent recurrent themes were adapted into a structured questionnaire to tap people's beliefs about the endurance of and changes in intimate relationships.

\subsubsection{Results and Discussions}

The qualitative study aimed to identify the relational beliefs in close relationships, specifically the nature and causes for changes in these relationships. Sixteen themes were frequently reported by different participants across the interviews; these themes were deemed important to the participants' perception of changes in relationships. Table 1 shows results of the theme coding of the content in the recorded transcripts, and their ranking in terms of frequency cited by participants.

The recurrent themes could be organized around three main perspectives on changes in relationships. As expected, changes as perceived by Singaporean 
Chinese included both induced and naturally occurring changes. As presented in Table 1, some of the most frequently mentioned beliefs concerning the nature of relationship-change revealed associations to the "agent" that instigates the changes, such as oneself, the environment and circumstances. For instance, item 1 and item 2 stated that relationship changes when the self or the environment changes respectively. This was followed by a second, frequently occurred theme, that changes are inevitable in life and uncontrollable as "people are unpredictable" (participants' words). For instance, item 8 referred to changes in relationships as "the only constant in life" (participants' words). This exudes a pragmatic outlook on the nature of relationships. Lastly, a third theme emerged suggesting that change in relationships is required as a form of maintenance to tackle the inevitable change or sometimes downturn in relationships (e.g., items ranking 10 and 11). Both partners in a relationship need to make positive changes for the sake of preserving the relationship (e.g., item 4).

The following are some examples of the interviewees' responses recorded and arranged in accordance with the three prevailing themes.

Who and what is responsible for change. When participants were asked about whether they think intimate relationships would change, some of the typical responses from the respondents were as follow:

Table 1. Coding frequency of recurrent themes.

\begin{tabular}{|c|c|c|}
\hline Rank & Themes & $\begin{array}{l}\text { Average } \\
\text { frequency }\end{array}$ \\
\hline 1 & Rps change when I change. & 98.5 \\
\hline 2 & Rps change due to environment or situation changes. & 34 \\
\hline 3 & Rps change as growth (part of growing process). & 22 \\
\hline 4 & $\begin{array}{c}\text { Rps change when we change together as a couple in the } \\
\text { relationship. }\end{array}$ & 20.5 \\
\hline 5 & Rps change due to being at different phases in my life. & 20 \\
\hline 6 & Rps change when I cannot stop my partner from changing. & 18 \\
\hline 7 & Rps change because people are unpredictable. & 13.5 \\
\hline 8 & Rps change as change is the only constant in life. & 13 \\
\hline 9 & Rps change due to being at different stages of relationship. & 13 \\
\hline 10 & $\begin{array}{l}\text { Rps change as a mean to compromise and work things out (e.g., } \\
\text { changing my perceptions). }\end{array}$ & 12 \\
\hline 11 & Rps change is to "preserve" the relationship from going downhill. & 8 \\
\hline 12 & Without progress, there will be deterioration. & 7 \\
\hline 13 & The longer the relationship, the more likely to change. & 4.5 \\
\hline 14 & $\begin{array}{l}\text { Rps change because people like to experience the highs and lows } \\
\text { in relationship, as the spice of life. }\end{array}$ & 4 \\
\hline 15 & $\begin{array}{l}\text { Rps change when my perception of my partner changes, after } \\
\text { being in the relationship for some time. }\end{array}$ & 4 \\
\hline 16 & Change as a deviation from expectation. & 4 \\
\hline
\end{tabular}

Note. Rps = Relationships. The Average Frequency referred to the average frequency count of quotes across the two raters that were coded as the listed theme. 
A male interviewee said:

Yes. As age goes by your thinking changes, then your concept of relationship changes ... If I started to mature, my thinking changes, so this may affect the relationship, but that is you making a change right?

Another female interviewee mentioned:

Yes, definitely, because humans will never be stagnant, that's how we survive through all these times, adapt and change to our environment ... change will come, definitely ... Mostly (in) the case (where) we do have some control over these changes, we can resist change, but I believe, ultimately, you'll still have to adapt to (the) environment. But the degree on how you adapt depends on yourself.

These responses highlight the dynamic nature of relationships: In relationships things might change, the changes can be people in the relationship, the environment and the natural progression of the relationship. These participants believe that the relationship will change as the partners or players of the relationship change.

Change is inherent in relationships. Participants frequently mentioned that change is inevitable. It is almost impossible to avoid or stop changes from occurring. This could be related to the unpredictability of human nature and the circumstances in which they live. The participants believed that human beings seek novelty and excitement in the relationship rather than stability or stagnation. Participants expressed the realistic views that one should not expect things to remain the same across time.

A male interviewee said:

I think it is still the same, but you can't really know what to expect from it (the relationship), so definitely I think it will still be changeable, because people are unpredictable, so don't really know what is going to happen ...

A female interviewee said:

... if I fall for a person, surely I hope it will last ... but forever? It depends. Any situation comes in and it may interrupt, maybe just split off ... so I am not sure. So I cannot have a concern that it may last forever. It may ... but it cannot, it will not ... something like that.

Another female interviewee also responded similarly:

... because there are so many things in life that will change, and these I think are out of your control. Let's say, one person decides to go overseas to work, another person decides to take (up) a new hobby ... you can't really say you are not working hard [on the relationship], but it's out of your control also.

Managing change. Participants, who mentioned the inevitability of change in relationships, suggested that deliberate effort to make changes in relationships is 
needed to keep the relationship from deteriorating. This theme seems to correspond to the traditional Chinese belief about learning, as mentioned by one of the interviewees, which proposes that one needs to continue putting in effort to maintain the current level of intellect and knowledge. Similarly, if no one introduces positive changes into the relationship, the relationship will be slipping downhill that may eventually lead to breaking up.

A female participant responded with "yes" to the question whether the relationship would change by itself if one made no attempt to change it. The inevitable changes are depicted as going "downstream", while effort are needed to maintain or strive for "upstream", she continued:

Because I remember there is a Chinese proverb that says that, if you do not continue to go upstream, you will go downstream. Can't remember ..., it's like (rowing a boat) upstream if you don't try to make the effort to go up stream, it will just float downstream.

Another female participant who shared a similar view said:

If I have a choice, I would make a change to the relationship. A relationship needs to be built up to a perfect one. So, if I don't make a change right, then this (the relationship) would never go on. So I think I will make a change to make a better one ... something like that.

A male interviewee commented on the topic whether relationships would change said:

It depends ... you can try to change certain things, or make an effort to improve or do differently. But I also don't really believe in trying to change people. I think for me (introducing change) is more like a matter of working something out, rather than trying to change things, more like come to a compromise. I guess it's like a change of expectations rather than (a) change of people's behavior.

Apparently the interviewee perceived that effort is needed to keep the relationship going, and this could involve proactive management or changing own expectations or in the form of making compromise in relationships.

It is noted when proactive changes are concerned, interviewees often mentioned "we" rather than "I" or "he/she". One male interviewee highlighted the importance of working together as a couple for "changes" to reap any benefits. He said,

... to maintain a relationship takes effort, both parties must actually work towards it ... Imagine if a couple just do their own thing ... there's no growth, there's no relationship, in fact it may even deteriorate ...

Male and female participants shared similar perceptions about changes in relationships. They perceived that inevitable change in relationships as caused by various agents: self, others and the situation, as well as the experiences connected to coming of age. Central to these beliefs is the underlying need to manage any 
possible change or deterioration that might occur as a natural part of relationship progression, by injections of positive changes to the relationships. This corresponds to the Chinese philosophical saying that: things would go downstream if one does not do something to "heave upstream" to prevent it (Liang, 1912, as cited in "Like a Boat Sailing", 2016). The changes identified by participants consisted of both natural change (i.e., changes that are inherent in relationships), and induced change (i.e., managing change in relationships).

The findings of this qualitative study contributed to the conceptualization of relationship beliefs in Singapore young adults. The 16 themes were adapted to construct the questionnaire "Relationship beliefs about change" for used in the large-scale quantitative study on relationship beliefs and attachment. Prior to that, the scale was subjected to validation in Study 2 via exploratory and confirmatory factor analysis.

\subsection{Study 2: Validation of Relationship Beliefs about Change}

\subsubsection{Participants}

An initial sample of four hundred and one participants was recruited for the validation study. The participants were undergraduate students of a major university in Singapore, who were enrolled in an introductory psychology course and obtained partial course credit for their participation. They were Singaporeans or permanent residents of Singapore, and all of them were ethnic Chinese. The ages of participants ranged from 18 to 29 years old ( $M=22.31$ for males, $S D=1.29$; and $M=20.31$ for females, $S D=1.07$ ), with 200 males and 201 females. Among them, 269 participants indicated that they were dating someone at the time of completing the questionnaire, or had intimate relationships in the past. There were 71 Christians, 12 Catholics, 191 Buddhists or Taoists, 1 Muslim, 123 free-thinkers and 3 from other religions. Ethical approval was obtained from the ethics committee of psychology division in the university institution.

Upon identifying the latent factors of RBC via exploratory factor analysis, confirmatory factor analysis was performed on another sample of three hundreds and twenty-five students in a psychology course in order to validate the factors found. There were 120 males and 205 females, age ranged from 18 to 29 years old ( $M=22.32$ for males, $S D=1.51$; and $M=20.16$ for females, $S D=1.15$ ). Similarly, only Singaporean Chinese who were dating or had dating experiences were recruited.

\subsubsection{Materials}

A demographic information page and a battery of scales were administered to the participants in the study. Five scales that are meaningfully related to relationships and implicit beliefs were employed to assess the construct and discriminant validity of the "Relationship beliefs" questionnaire.

Relationship beliefs about change ( $R B C$ ). The scale was developed based on the qualitative data collected from the semi-structured interviews conducted earlier. The scale consisted of 16 recurrent themes obtained from the interview 
study, for instance, "Relationships change as people are unpredictable". The scale aimed to assess participants' perspective about change in close relationships, particularly dating relationships. The term "change" was preferred over "malleable" in the questionnaire as this was the term most participants iterated during interviews, and "change" would be a more comprehensive term that might encompass more than the concept of malleability, including changes brought about by time (temporal changes). Participants were instructed to respond according to their beliefs and expectations about changes in emotionally intimate relationships. Participants were required to indicate their responses on a Likert scale, ranging from 1 to 7 , with higher scores indicating higher perceived change in relationships. The questionnaire obtained reliability alpha of .80 for the overall 16 -item scale.

Experience in close relationships-revised (ECR-R). (Fraley, Waller, \& Brennan, 2000). The ECR-R is a 36-item self-report measure on adult attachment. The scale assesses the rating of an individual's attachment style on two dimensions, namely anxiety and avoidance. Each dimension has 18 items, to be scored on a Likert scale, ranging from 1 to 7 , with higher scores indicating higher levels of insecure attachment on the respective dimension.

The scale obtained in earlier studies an internal reliability of alpha 0.95 and 0.93 for anxiety and avoidance sub-scales respectively (Fraley et al., 2000). However, Fraley et al., (2000) mentioned the limitation of the scale's fidelity in measuring lower levels of insecure attachment as opposed to higher levels. Sample items for the anxiety dimensions are "I'm afraid that I will lose my partner's love", and "I find that my partner(s) don't want to get as close as I would like". Items for the avoidance dimension include "I get uncomfortable when a romantic partner wants to be very close" and "I find it difficult to allow myself to depend on romantic partners". The internal reliability obtained for the current study was high, at .90 for the anxiety dimension and .87 for the avoidance dimension.

Implicit beliefs about intelligence (IBI). (Dweck, Chiu, \& Hong, 1995). This questionnaire was chosen for exploring discriminant validity of the RBC scale. The IBI scale is based on implicit beliefs about human attributes, specifically intelligence, and assesses whether one believes that intelligence is a trait-like fixed attribute that cannot be changed (i.e., entity theory), or is malleable and can be improved (i.e., incremental theory). Although it measures similar properties as $\mathrm{RBC}$ regarding malleability, it targets the attribute of intelligence, which is a distinctively different attribute from relationship beliefs. It is hence expected that this scale will show only small or no association with RBC.

The scale consists of only three items, for example, "Your intelligence is something about you that you can't change very much". Participants would respond on a continuous scale ranging from 1 to 7 . The scores are reverse-coded such that higher scores indicate greater endorsement of the incremental theory. Reported alpha for the IBI scale ranged from .94 to .98 in previous studies (Dweck, et al., 1995), and an alpha of .89 was obtained for the current study. 
Implicit theories of relationships (ITR). (Knee, Patrick, \& Lonsbary, 2003). The ITR scale is a 22-item questionnaire that assesses the extent to which one subscribes to "destiny belief" and/or "growth belief" in romantic relationships, with each belief consisting of 11 items. Destiny belief refers to the belief of whether partners in the relationship are compatible and meant to be together, for instance, "Unsuccessful relationships were never meant to be", and "Relationships that do not start off well inevitably fail". On the contrary, growth belief refers to the belief that relationship problems can be overcome if one puts in the effort, for instance "With enough effort, almost any relationship can work", and "Challenges and obstacles in a relationship can make love even stronger". The ITR is measured on a 7-point Likert scale, with higher scores indicating greater adherence to the respective belief dimension. The reported alpha for destiny and growth beliefs were .82 and .74 respectively, and scores of the two beliefs are found to be independent of each other (Knee, et al., 2003). Internal reliabilities of the destiny and growth beliefs obtained for the current study were .84 and .75 respectively.

Relationship beliefs inventory (RBI). (Eidelson \& Epstein, 1982). The RBI consists of five subscales that measure five different types of dysfunctional beliefs in romantic relationships. The five dysfunctional beliefs include expecting partners to read each other's mind, disagreement among partners are considered as a threat to loving relationships, believing that partners are unable to change themselves or the relationship, expecting partners to be perfect sexual partners, and stereotypical thinking about the differences among men and women. Each subscale has 8 items, to be administered on a continuous scale ranging from 1 to 7 , with higher scores indicating greater adherence to the belief. In an effort to shorten the battery of scales while maintaining sufficient information for validation, only two of the subscales, namely "Partner cannot change" and "Disagreement is destructive", were selected for the current validation study. Reported alpha coefficients for "Partner cannot change" and "Disagreement is destructive" were .72 and .81 respectively (Eidelson \& Epstein, 1982). Internal reliabilities obtained for the current study were .62, and .80 for the two selected scales, respectively.

Relationship assessment scale (RAS). (Hendrick, 1988; Hendrick, Dicke, \& Hendrick, 1998). The RAS measures the extent to which an individual is happy with the relationship for both marital and dating relationships. Since this is a measure of satisfaction toward the couple relationship, only 269 (instead of the total 401) participants with past or current experience in intimate relationships were included in the analysis. It consists of 7 items, to be scored on a 7-point Likert scale, with higher scores indicating higher levels of satisfaction, among which item 2 and 7 are coded in the reverse direction. Sample items include "How good is your relationship compared to most?" and "To what extent has your relationship met your original expectations?” Reported item-total correlation ranged from .57 to .76 , with an alpha of .86 (Hendrick, 1988). The scale was selected to examine the predictive validity of the RBC scale in the current study. Internal reliability obtained for the current sample was .86 . 


\subsubsection{Procedures}

All participants completed a battery of questionnaires including Relationship Beliefs about Change (constructed for the present study by the current authors), Experience in Close Relationships-Revised (Fraley, et al., 2000), Implicit Beliefs about Intelligence (Dweck, et al., 1995), Implicit Theories of Relationships (Knee, et al., 2003), two subscales of Relationship Belief Inventory (Eidelson \& Epstein, 1982), and Relationship Assessment Scale (Hendrick, 1988; Hendrick, et al., 1998). Participants were undergraduates in a local university enrolled in an introductory psychology course, whereby participation in the study would contribute partial credit to the course. Participants were required to complete the questionnaires within 30 minutes on campus.

\subsubsection{Results}

Participants' mean responses on the scales are summarized in Table 2. The data were first examined for mean differences on the demographic variables that are often regarded to have implications on relationship beliefs.

\section{Gender, Religion and Past relationships}

Participants' relationship beliefs about change were assessed for differences across gender, religious background, and whether they have had experience of being involved in intimate relationships. Independent samples $t$-test was performed on gender and prior or current experiences with intimate relationships; ANOVA was performed across participants' religious affiliations. There were no significant mean differences across gender, $t(399)=.385$, ns; and no significant differences across dating experiences $t(399)=1.905$, ns. Similarly, no significant

Table 2. Mean responses of RBC and validation scales.

\begin{tabular}{ccc}
\hline Scales & $M$ & $S D$ \\
\hline RBC & 5.00 & 0.62 \\
IBC & 3.96 & 1.47 \\
Destiny Beliefs-ITR & 3.79 & 0.87 \\
Growth Beliefs-ITR & 5.01 & 0.65 \\
Disagreement-RBI & 3.19 & 0.80 \\
Partner Cannot Change-RBI & 3.31 & 0.63 \\
Anxiety-ECR-R & 3.57 & 0.93 \\
Avoidance-ECR-R & 3.01 & 0.72 \\
RAS & 5.06 & 0.91 \\
\hline
\end{tabular}

a. $\mathrm{N}=401$ (except for RAS which assesses the level of satisfaction in past intimate relationships, $\mathrm{n}=269$, whereby only 269 out of 401 participants have experience in having intimate relationships with a partner), b. $\mathrm{RBC}=$ Relationship Beliefs about Change; IBI = Implicit Beliefs about Intelligence; Destiny Beliefs-ITR = Destiny dimensions in Implicit Theories of Relationships; Growth Beliefs-ITR = Growth dimension in Implicit Theories of Relationships; Disagreement-RBI = Disagreement subscale of Relationship Beliefs Inventory; Partner Cannot Change-RBI = Partner Cannot Change subscale of Relationship Beliefs Inventory; Anxiety-ECR-R = Anxiety dimension of Experience in Close Relationship-Revised; Avoidance-ECR-R = Avoidance dimension in Experience in Close Relationships-Revised; and RAS = Relationship Assessment Scale. 
mean differences were found across different religious background, $F(5,395)$ $=.674$, ns. It appears that relationship beliefs about change are held similarly among Chinese men and women as a fundamental attitude espoused towards life and relationships. Since it is a commonly shared belief, which is likely to develop from a young age, it does not require prior experiences in romantic relationships, nor has it been significantly affected and differentiated by such experience.

\section{Exploratory Factor Analysis (EFA)}

The internal structure of the scale was assessed in two steps. Exploratory factor analysis was first performed on the validation dataset to examine for underlying dimensions of the scale. This was followed by confirmatory factor analysis using a new set of data collected subsequently for validation.

Data screening found 16 multivariate outliers that were removed from the analysis, resulting in 385 cases. Preliminary analysis showed that the scores have good factorability: the Kaiser-Meyer-Olkin Measure of Sampling Adequacy was .79, and Bartlett's Test of Sphericity was significant at $p<.001$. In an attempt to examine the underlying dimensions of the scale, principal component analysis was performed on the 16-item RBC scale. An initial component extraction showed that there were five dimensions with eigenvalues greater than one. However, on observing the Scree plot, the amount of variance each factor accounted for and considering the meaning of the dimensions, principal component analysis was re-run with Promax rotation, extracting three components.

As presented in Table 3, the first, second and third dimensions accounted for $26.58 \%$ (eigenvalues $=4.25), 10.86 \%$ (eigenvalues $=1.74$ ) and $8.92 \%$ (eigenvalues $=1.43$ ) of the total variance respectively. The component loadings are presented in Table 4. Utilizing a cutoff point of .40, there were seven items loaded under the first component extracted, five items loaded under the second component, and three items loaded under third component. It was noted that there was one item (i.e., item 16) that failed to load under any dimensions given the cutoff point. This resulted in a 15-item scale after removing item 16 .

The questionnaire, Relationship Beliefs about Change was constructed to assess beliefs about the endurance and consistency in intimate relationships. The variables loaded under first dimension appeared to refer to the agents that might initiate change in intimate relationships, such as, "relationships change when I change", "relationships change when environment or situations change", and "relationships change as it moves from stage to stage". Hence, Factor 1 is named

Table 3. Eigenvalues and variance accounted by the components obtained from PCA.

\begin{tabular}{cccc}
\hline & \multicolumn{3}{c}{ Components } \\
\cline { 2 - 4 } & 1 & 2 & 3 \\
Eigenvalues & 4.25 & 1.74 & 1.43 \\
Variance & $26.58 \%$ & $10.86 \%$ & $8.92 \%$ \\
\hline
\end{tabular}

a. $\mathrm{PCA}=$ Principla Component Analysis. 
Table 4. Component loadings for the relationship beliefs about change scale.

\begin{tabular}{|c|c|c|c|c|}
\hline No. & Scale Items & $\mathrm{C} 1$ & $\mathrm{C} 2$ & $\mathrm{C} 3$ \\
\hline 1 & Relationships change when I change. & .82 & -.22 & .00 \\
\hline 3 & Relationships change as individuals grow. & .73 & -.05 & .09 \\
\hline 5 & Relationships change when my partner changes. & .72 & -.10 & -.03 \\
\hline 4 & $\begin{array}{l}\text { Relationships change when I am at different phases of my } \\
\text { life. }\end{array}$ & .71 & -.01 & .00 \\
\hline 2 & Rps change when environment or situation changes. & .71 & .08 & -.09 \\
\hline 8 & Relationships change as it moves from stage to stage. & .43 & .2 & .20 \\
\hline 15 & $\begin{array}{l}\text { Relationships change when the relationship is not what I } \\
\text { expected. }\end{array}$ & .43 & .31 & -.10 \\
\hline 12 & The longer the relationship, the more likely it will change. & -.22 & .71 & .03 \\
\hline 13 & $\begin{array}{l}\text { Relationships change because people like to experience the } \\
\text { highs and lows in relationship, as the spice of life. }\end{array}$ & -.05 & .68 & -.20 \\
\hline 14 & $\begin{array}{l}\text { Relationships change when my perception of my partner } \\
\text { changes after being in the relationship for some time. }\end{array}$ & .30 & .64 & -.12 \\
\hline 6 & Relationships change as people are unpredictable. & .08 & .47 & .21 \\
\hline 7 & Relationships change as change is the only constant in life. & -.10 & .42 & .31 \\
\hline 10 & $\begin{array}{l}\text { Relationships change to keep the relationship from going } \\
\text { downhill. }\end{array}$ & -.10 & -.14 & .84 \\
\hline 9 & $\begin{array}{l}\text { Relationships change as a mean to compromise and work } \\
\text { things out between partners. }\end{array}$ & .11 & -.09 & .75 \\
\hline 11 & $\begin{array}{c}\text { Without progress, things will deteriorate eventually, so as } \\
\text { relationships. }\end{array}$ & -.05 & .35 & .49 \\
\hline 16 & Relationships change when we change together as a couple. & .33 & .04 & .33 \\
\hline
\end{tabular}

a. Loadings $>.40$ are in boldface.

"Agent of Change" (i.e., AGC). It is apparent that Singaporean Chinese perceive changes in relationships can arise from the self or the partner, the relationship itself, and/or the environment or context. When any of these attributes change, the relationship loses its consistency.

On the other hand, the five items in the second dimension described participant's belief about the inevitability or necessity of change in intimate relationships, such as "the longer the relationship, the more likely it is to change", "relationships change as people are unpredictable", and "relationships change as change is the only constant in life". This second factor describing the realistic perspective of changing people and world is named "Inevitable Change" (i.e., IC), as the items seem to suggest that relationships will inevitably change given sufficient time.

The third dimension consisted of three items, which put forth the perspective that change is needed in order to maintain or salvage relationships. This includes items such as "relationships change to keep relationships from going downhill" or "relationships change as a means to compromise and work things out between partners". In other words, it seems that the beliefs about change involve the belief that changes in relationships are needed to keep the relationship going. Without any intervention, the relationships are likely to deteriorate. Component 
2 assesses realistic perceptions about inevitable change in relationships, while Component 3 assesses the perspective that some changes might bring about positive outcomes to deal with the ever-changing romantic relationships. Hence, this third factor is named "Managing Change".

Factor correlations are presented in Table 5. All three dimensions were significantly correlated with each other. The positive correlations suggest that changes in relationships are more likely to be perceived as inevitable if more changes are expected of the relevant agents of change, such as the partner. Correlation between Factor 2 and 3 suggests that the more one expects the relationship to change, the more effort to manage the relationship is expected.

The overall RBC scale demonstrated reasonable internal reliability for 15 items at $\alpha=.79$. Alpha coefficients obtained for each subscales were as follows: Agents of Change ( $\alpha=.79$ for 7 items), Inevitable Change ( $\alpha=.60$ for 5 items), Managing Change ( $\alpha=.62$ for 3 items).

\section{Confirmatory Factor Analysis (CFA)}

As reported above, the $\mathrm{RBC}$ scale was constructed using recurrent themes reported by interviewees. EFA results reported earlier have identified three dimensions of the RBC scale, indicating that the beliefs about changes in relationships are fundamentally about (1) who or what makes the changes, (2) changes are inevitable, and (3) change management. Hence, the three dimensions are accordingly named (1) Agent of Change (i.e., who or what is essentially responsible for the change), (2) Inevitable Change (i.e., natural changes or downturns in relationship that is inevitable temporally or in accord with human nature), and (3) Managing Change (i.e., making changes to sustain the probable deterioration of relationships).

In order to obtain support for this three-dimensional structure of $\mathrm{RBC}$ observed in the exploratory factor analysis, CFA was conducted with a new dataset as a validating sample. Data screening was performed and 13 multivariate outliers were deleted from the data, resulting in a sample size of 312 . Some items from the original scale were modified for clarity after receiving feedback from participants and considering their low communalities. For instance, "Relationships change because people like to experience the highs and lows in relationships, as the spice of life", was shortened to include only "Relationships change because people like to experience highs and lows in relationships". Commonly reported fit indices like the Chi-square $\left(\chi^{2}\right)$, the comparative fit index (CFI), the

Table 5. Correlations among the factors of relationship beliefs about change.

\begin{tabular}{clccc}
\hline & & \multicolumn{3}{c}{ Factors } \\
\cline { 3 - 4 } & Factors & 1 & 2 & 3 \\
\hline 1 & Agent of Change & - & $.37^{\star *}$ & $.33^{\star *}$ \\
3 & Inevitable Change & $.37^{\star *}$ & $-32^{\star *}$ & - \\
\hline
\end{tabular}

a. ${ }^{* *} p<.01$. 
incremental fit index (IFI), and the root mean square error of approximation (RMSEA) were utilized to evaluate the fit of the model. The present study adopted the criteria that a model is considered to have good fit if CFI and IFI exceed .90; while the RMSEA to be near .06 within a $90 \%$ confidence interval (Chen, Curran, Bollen, Kirby, \& Paxton, 2008; Hu \& Bentler, 1999; MacCallum, Browne, \& Sugawara, 1996).

Results of the fit indices from the initial run of CFA indicated moderate unsatisfactory fit of the model on the new data $\left(\chi^{2}(87)=265.19\right.$, CFI $=.76$, IFI $=.77$, RMSEA $=.08)$. However, all paths coefficients estimated in the model were statistically significant at $p<.01$, standardized loadings ranging from .20 to .76 across the three factors. Large modification indices (i.e., MI) suggest the possibility for substantial content overlapping (Byrne, 2010). Two pairs of error terms that had much larger MI than the rest of the pairs of error terms were: error terms of item 1 and 5, and error terms of item 15 and 14.

On observing that item 14 "Relationship change when my perception of my partner changes after being in the relationship for some time" seems to be a specific example of and provides clearer explanation for item 15 "Relationships change when the relationship is not what I expected", it was decided to drop item 15 from the model. A closer scrutiny of item 1 and 5 revealed that the two items are meaningfully related, with item 1 referring to change in the self, while item 5 refers to change in the partner. Romantic relationships involve two very closely related persons, and the beliefs about change in the relationship essentially include beliefs about change in both parties. Therefore, error terms of item 1 and 5 were allowed to correlate and CFA was rerun on the 14-item scale. Results showed much improved fit with AIC reducing from 331.19 to $273.14\left(\chi^{2}\right.$ $(73)=163.60$, CFI .87 , IFI $=.87$, RMSEA $=.06)$. The fit was acceptable though not excellent.

A new exceptionally large MI was found between the error term of item 1 and 3. Item 3 "Relationships change as growth" apparently overlapped in meaning with item 1 "Relationships change when I change" for young adults. Hence, to further improve the fit of the model, these two error terms were allowed to correlate. The resulting model indicated satisfactory fit with $\chi^{2}(72)=135.34$, CFI $=.91$, IFI $=.91$, RMSEA $=.05$. Significant change in chi-square $\left(\Delta \chi^{2}(1)=28.26\right.$, $p<.01)$, revealed that the model with more paths better represented the beliefs about change in relationships. All latent factors were significantly correlated, and all parameters estimated in the three-dimensional model of RBC were significant at $p<.01$, with standardized coefficients ranging from .20 to .78. Figure 1 shows the resulting model with 14 items, and two pairs of related error terms.

\section{Convergent, Discriminant and Predictive Validity}

Convergent validity of the scale is demonstrated when the scale has moderate to high correlations with measures of conceptually related constructs; and discriminant validity is observed if the scale has low or no correlation with measures of less or non-related constructs. Correlations were computed to examine the $\mathrm{RBC}$ scale for convergent and discriminant validity. The correlations are re- 
ported in Table 6.

One of the validating scales, the IBI, measures the beliefs about whether intelligence is fixed or malleable. As expected, there was a significant but low relationship between IBI and Inevitable Change ( $\mathrm{r}(399)=.17, p<.01)$, and between IBI and Agent of Change ( $\mathrm{r}(399)=.13, p<.01$ ), suggesting some overlapping facets assessed by the two scales as both refer to people's underlying beliefs about the changing reality. However, there was no significant relationship between IBI and Managing Change, providing discriminant validity for RBC as a measure of implicit beliefs in the relationship domain. The Managing Change facet suggests that people make changes to the relationship in order to deal with the perceived inevitable and deteriorative change; this distinguishes RBC from other implicit beliefs such as the IBI.

The ITR scale assesses whether individuals endorse destiny beliefs or growth beliefs about romantic relationships. People who adhere to destiny beliefs would



Figure 1. Confirmatory factor analysis on relationship beliefs about change. All paths were significant at $p<.01$. Fit indices were $\chi^{2}(72)=135.34$, CFI $=.91$, IFI $=.92$, RMSEA $=.05$. 
Table 6. Correlations among RBC, IBI, RBI, ECR-R, and RAS.

\begin{tabular}{cccc}
\hline \multirow{2}{*}{ Scales } & \multicolumn{3}{c}{ Relationship Beliefs about Change } \\
\cline { 2 - 4 } & Agent of Change & Inevitable Change & Managing Change \\
\hline IBI & $-.13^{* *}$ & $-.17^{* *}$ & -.07 \\
ITR (Destincy Beliefs) & .08 & $.26^{* *}$ & .00 \\
ITR (Growth Beliefs) & $.21^{* *}$ & $.16^{* *}$ & $.39^{* *}$ \\
RBI (Disagreement) & -.02 & .11 & $-.15^{* *}$ \\
RBI (Partner Cannot Change) & -.09 & .09 & $-.27^{* *}$ \\
ECR-R (Anxiety) & .06 & $.19^{* *}$ & -.07 \\
ECR-R (Avoidance) & -.11 & .05 & $-.24^{* *}$ \\
RAS & .02 & -.11 & $.23^{* *}$ \\
\hline
\end{tabular}

a. $\mathrm{N}=401$ (except for Satisfaction, $\mathrm{N}=269$, due to the exclusion of those who have never been involved in romantic relationships); b. IBI = Implicit Beliefs about Intelligence; ITR = Implicit Beliefs about Relationships; RBI = Relationship Beliefs Inventory; ECR-R = Experience in Close Relationship-Revised; RAS = Relationship Assessment of Satisfaction. ${ }^{* *} p<.01$.

think that successful relationships rely on finding the ideal partner; while people who subscribe to growth beliefs think that good relationships are cultivated and developed. The significant correlations between RBC scale and the ITR scale supported the validity of the RBC. A closer look revealed that only the Inevitable Change dimension was positively related to Destiny Beliefs in the IBC ( $\mathrm{r}$ (399) $=.26, p<.01$ ), but not Agent of Change or Managing Change. This was expected, as destiny beliefs focus on finding an ideal fit in relationships rather than working on the relationship to achieve a fit. It is likely that people who subscribe to destiny beliefs do not think that there is a need to work on the relationship to sustain it. If the partners were compatible, things would go smoothly. Conversely, the dimension of growth beliefs was significantly related to all three dimensions of the RBC. The association between growth beliefs and Managing Change was especially substantial $(\mathrm{r}(399)=.39, p<.01)$ as both factors involve intentional effort in supporting or developing a stable relationship, regardless of underlying differences in the expectation of relationships' nature and progress. In contrast, Agent of Change and Inevitable Change showed weaker correlations with growth beliefs $(\mathrm{r}(399)=.21 p<.01$ and $\mathrm{r}(399)=.16, p<.01$ respectively). It is noted that growth beliefs entail the perspective that effort could improve a less than ideal relationship, which partly overlaps with the Managing Change belief on the proactive effort deemed necessary to maintain or develop a relationship.

$\mathrm{RBC}$ was also examined for its relationship with another existing scale about relationship beliefs, the RBI scale. Among the two subscales of the RBI, Partner Cannot Change showed moderate relationship with MC ( $\mathrm{r}(399)=-.27, p<.01)$, Disagreement is Destructive showed significant but low association with MC ( $\mathrm{r}$ 
$(399)=-.15, p<.01)$. Both subscales of the RBI were unrelated to the other two dimensions of RBC (i.e., Agent of Change and Inevitable Change). Results revealed that $\mathrm{RBC}$ can be distinguished from other relationships' measures that tap into different aspects of relational beliefs. The negative relationship between Partner Cannot Change and Managing Change, and between Disagreements is Destructive and Managing Change, suggests that maladaptive beliefs about one's partner are negatively related to one's effort to maintain or salvage a deteriorating relationship. Hence, results showed some overlapping facets measured by Managing Change, Partners Cannot Change, and Disagreement is Destructive; while Agent of Change and Inevitable Change are independent of the two subfactors of RBI.

Further validation was provided by associating the ECR-R scale with the RBC. Attachment anxiety was found to be positively related to Inevitable Change ( $\mathrm{r}$ $(399)=.19, p<.01)$ but not related to the other two factors of RBC; while attachment avoidance was negatively related to Managing Change ( $\mathrm{r}(399)=-.24$, $p<.01$ ), but did not correlate with Agent of Change and Inevitable Change of the RBC scale. A realistic outlook on the ever-changing nature of relationships appeared to be associated with attachment anxiety, and people with avoidant style of attachment seemed to believe that any effort to change is unlikely to salvage the deteriorating relationships. Indeed, attachment-related patterns differ across individuals owing to the type and compositions of the internal working models of attachment (Collins, Guichard, Ford, \& Feeney, 2004) that individuals held. The two dimensions of adult attachment are indeed varying combinations of working models of self and others (Collins, et al., 2004). Since results demonstrated that Agent of Change and Managing Change were independent of Anxiety, suggesting that anxious attachment does not necessarily lead to inertia or maladaptive patterns of relationship behavior. Furthermore, Inevitable Change and Managing Change were independent of Avoi- dance. This suggests that these beliefs might account for areas that the working model of "self" and "other" in attachment did not manage to capture. However, subsequent research is needed to establish these possibilities.

Relationship Assessment Scale (RAS) measures how much a person is satisfied with his or her relationship. The dimension of Managing Change of RBC was found to be significantly associated with RAS. Participants who were high in Managing Change were more likely to be satisfied with their relationship. This provided predictive validity to the $\mathrm{RBC}$. Overall, the results demonstrated acceptable properties of the RBC scale in terms of convergent, discriminant and predictive validity through examining the relationships between RBC scale and other related relationship scales.

In sum, Study 1 and 2 were mainly exploratory studies aimed at understanding the young people's beliefs about changes in romantic relationships, as well as to construct a detailed assessment of such beliefs. The validation study examined the psychometric properties of RBC scale by examining the underlying structure of the scale. The three dimensions of RBC were first identified via EFA, and were 
supported via CFA on a second dataset. CFA results showed that the three-dimensional structure of RBC explained the current data well, and that the dimensions were moderately related to each other. The relationships between the three dimensions of $\mathrm{RBC}$ and various close relationship scales, or measures about the perspective of change, were examined for convergent and discriminant validity.

Apparently, the three facets identified in the factor analysis, namely Agent of Change, Inevitable Change, and Managing Change, represent people's beliefs about change in intimate relationships. It seems that Singaporean Chinese believes that relationships are bound to change if they are left to run their own course. Change may stem from either party in the relationship, the inevitable changes that result from a relationship's natural growth and development, and the changing environment and perceptions of the partners. However, the presence of Managing Change as a distinct factor suggests that it is this belief of inevitable change that motivates Chinese to introduce changes or put in effort to delay or stop relationships from deteriorating.

This new multidimensional construct of change beliefs could be understood as a cognitive representation of relationship consistency and stability that supplement our knowledge about the representations of self and others as described in attachment studies (e.g., Pietromonaco \& Barrett, 2000). Furthermore, this relationship representation is intimately related to the self-and-others cognitions, which could lead to important consequences on relationship outcomes.

\section{General Discussion}

The first objective of the present study was to explore young adults' conception of close relationships in regard to the perceived changeability of the intimate relationships. This was achieved through qualitative interviews with Singaporean Chinese university students in Study 1. Secondly, we constructed a measure to capture indigenous relationship beliefs and the expectation about change in intimate relationships. A total of 16 items were derived from the recurring themes obtained from the semi-structured interviews in Study 1. The newly constructed scale was validated in Study 2 and demonstrated acceptable psychometric properties. Exploratory factor analysis was performed on Singaporean Chinese undergraduates, and three meaningful dimensions within the Relationship Beliefs about Change (RBC) questionnaire were found. They are "Agent of Change", "Inevitable Change", and "Managing Change", and this three-factor model was confirmed via CFA with a second dataset obtained from the same university with another group of Singapore Chinese students. The resulting 14-item questionnaire (see Appendix) revealed that change beliefs in the local Chinese formed a multi-faceted, coherent system of beliefs that includes perceptions about who and what is responsible for changes in the relationship, believing in inevitable changes, and asserting effort to manage the change in order to maintain the relationship. Overall, the validation results showed support for this three-dimensional $\mathrm{RBC}$ scale as a measure of change beliefs in intimate relationships. 


\subsection{The Construct of Relationship Beliefs about Change}

The RBC scale is constructed based on lay people's conceptualization and expectations about changes in the romantic relationship. Its multidimensional content is comprehensive in explaining individual's beliefs of relationship change covering agent of change, direction and inevitability of change, as well as managing change. As expected, the change beliefs and perceptions revealed in semi-structured interviews include both induced and natural changes. Examples of interview responses that revealed natural change include "... because there are many things in life that will change, and these I think are out of your control ...." indicating the changes that are beyond the individual's power to control. On the other hand, interview responses towards whether relationship would change, also included instances that refer to induced change, such as "... if I don't make a change right, this [the relationship] would never go on. So I think I will make a change to make a better one ...", This is consistent with Rogg and Bradbury's (2002) notion of differentiating induced change, or changes made by intentional effort, and natural change, or changes that occur without purposive intervention, and that both are essentially part of the dynamic of the changing reality.

Similarly, responses revealed in the qualitative interviews as wells as items across the three dimensions revealed in EFA and CFA demonstrated both natural and induced change as perceived in intimate relationships. For instance, in the dimension of Agent of Change revealed in EFA, items such as "Relationship change when I am at different phases of my life", and "Relationship change when it moves from stage to stage" depicted perception of relationship change as a form of natural process rather than deliberate effort. On the other hand, items in the dimension of Managing Change such as "Relationship change to keep the relationship from going downhill” implied effort is required to maintain or salvage ongoing relationships.

However, this is in contrast to past research on implicit beliefs about relationships and other attributes, which focused mainly on effort to change (e.g., Canevello \& Crocker, 2011; Knee, 1998; Dweck, et al., 1995). Dweck, et al., (1995) provided important insights to implicit beliefs about change in various human attributes; however, their concept of change focused on a single dimension of entity versus incremental beliefs on human attributes. Canevello and Crocker (2011) discussed the predictors leading to the endorsement of growth beliefs, which resulted in better outcomes. These researches are mainly concerned about malleability or more precisely, the ability to change. The present study obtained insights on young people's conceptions of potential changes in intimate relationships, which was found to include essentially both the possibility of induced change or malleability, and the natural changes that occur over time. In this way, the study extended the notion of change by identifying naturally occurring changes in addition to deliberate change in relationships.

This notion of change in close relationships perceived by the young adults in Singapore is unique in the first dimension of RBC, "Agent of Change", which 
indicates the belief that the self, partner, environmental factors, relationship process and even natural growth or maturity of relationships are responsible for changes in intimate relationships. When any of these agents change, the relationship will change correspondingly. This highlights the dynamics of relationships, including the course of relationship, which is vulnerable to any changes bring forth by these stated agents. Apparently, not only the self and relationship partner are important factors, the contextual factors (e.g., "relationships change when environment or situations change") and the natural process of development (e.g., "relationships change when I am at different phases of my life", "relationships change when it moves from stage to stage") that are often beyond the direct control of individuals, are also critical to relationship beliefs about change. This emphasis on situational factors is influenced by the larger cultural social niche, in which the relationship grows and develops. This is again consistent with Rogg and Bradbury's (2002) conceptualization of multifaceted change. This contextual emphasis further reinforces the findings on cultural differences in cognitive styles (for instance, Nisbett, 2003; Choi, Nisbett, \& Norenzayan, 1999) that East Asians tend to attribute cause of events to external or contextual variables rather than internal variables as compared to the western counterparts. Paradoxically, the Chinese would take the responsibility to manage the relationship, which is consistent with the findings in a recent study on the cognitive flexibility and psychological resilience of the elderly Singaporeans (Chang, Toh, Chan, \& Fan, 2015).

Singaporean Chinese are collectivistic like many East Asians under the cultural influences of Confucianism (Ji, et al., 2010). Comparing to people from individualistic societies, individuals from the relatively collectivistic cultures tend to place emphasis on external forces in their social relationships (Leung, 1996). It is apparent from the RBC scale that both, the couple and the circumstances, are cited as the agents that initiate or contribute to changes in romantic relationships. Chan, et al., (2010) reviewed that the role of contextual or external factors is what differentiated Chinese perspectives on relationships from their western counterparts. This is in line with the present study, whereby the Chinese participants conceptualized relationship change to include contextual factors as an essential part in the natural development of relationships, for instance, "relationship change when environment and situation change". Similarly, in Chang and Chan's (2007) study, they obtained empirical support that circumstantial factors and opinions or inputs from the social network were deemed as important as intrapersonal and dyadic perspectives on relational decisions, such as whether it is the right time to get married. In similar way young adults in Singapore value the needs and the perspectives of their significant others or their social community (Chang, \& Lee, 2012). This coincides with the past studies that revealed interdependent relationships has important consequences for allocentric individuals' psychological adjustment and health including children (e.g., Schaerfl, 2009), and impact on romantic relationship passion and commitment (Bejanyan, Marshall, \& Ferenczi, 2015). 
In a nut shell, the findings from the present study are consistent with the notion of multi-faceted changes that includes both induced change and natural change (Rogge \& Bradbury, 2002). Additionally, relationship researcher, Berscheid (2010) contended that intimate relationships are bound to change when the environment that informs the relationship, changes with time. In this way, our participants perceived relationship change as inevitable, in that things might change for better or worse.

Therefore, changes in romantic relationships might occur when critical agents such as the self, the partner, environment, situation and relationship process are involved. Moreover, this perspective of change is realistic and pragmatic, rather than idealistic, as revealed in "Inevitable Change". For instance, items such as "the longer the relationship, the more likely it will change", "relationships change as people are unpredictable" and "relationships change as change is the only constant in life", share the perceived uncertainty of the state and direction of the relationship in the future. They exude the underlying anxiety about the potential change in romantic relationships. Furthermore, items in the dimension of Managing Change such as "Without progress, things will deteriorate eventually, so as relationships" also implies a realistic attitude that at times, relationship can deteriorate and not necessarily always changing for the better. In other words, intimate relationships are bound to change and may even deteriorate if allow to run their own course. Past studies have differed by proposing change beliefs predominantly as having positive implications on relationship outcomes (e.g., Knee, et al., 2003). Change beliefs are often associated with the ability to make positive changes or simply referred as the potential of growth and development in individuals (e.g., Dweck et al. (1995) entity versus incremental beliefs; and Eidelson \& Epstein's (1982) "Partner cannot change" dimension in "Relationship Belief Inventory”). However, Vangelisti, Reis, \& Fitzpatrick's (2002) study on the stability and change in romantic and marital relationships conceptualized changes as unavoidable or sometimes deterioration of the relationship. This corresponds to the expectation of potential deterioration embedded in RBC. In the present study, participants held a more realistic and multifaceted view about relationship change whereby change can occur for the better or worse.

Nevertheless, this multifaceted outlook (which includes induced and natural change) might serve as the motivating force for the Singaporean Chinese in dealing with possible changes in intimate relationships. This is evident in the "Managing Change" dimension of the $\mathrm{RBC}$ scale. "Managing Change" hinges on the realistic outlook about the unavoidable and constantly changing reality of intimate relationships (see item 11 in Table 4), and perceives relationship change as a proactive effort to curb, if any, potential deterioration (see item 9 and 10 in Table 4) of the relationship. This seems to be consistent with the proposition that the Chinese hold beliefs about change as non-linear or perhaps cyclical (Ji, Nisbett, \& Su, 2001; Ji, et al., 2010) whereby change might initiate or indicate both progression and deterioration in relationships at different point of time or 
from different point of view. With this paradoxical view, the Chinese might expect more changes to come, whether in good or bad times. The Chinese are carefully reflective toward most events and are inclined to anticipate change (Ji, et al., 2001). This means that Chinese individuals expect change regardless of whether the present state is satisfactory or not. When things are going well, they anticipate potential deterioration; when things are not going well, they expect it to change for the better. In this way, the Chinese are often vigilant about the potential changes, and are constantly preparing for potential down turn of events (Chang \& Sivam, 2004).

From the RBC scale, it is apparent that although young adults see changes in intimate relationships as inevitable and at times, unpleasant or undesirable, they nevertheless believe that proactive effort is needed to deal with the changes over time. This deliberate intervention is by itself another form of change. This mindset admonishes the relationship partners to be mindful to relationship changes and to stay vigilant during both good and bad times. Similarly, there are strong social norms in collectivistic societies for individuals to observe their obligations and roles in order to achieve harmony (Goodwin \& Tang, 1996). However, this is not a passive observation but an earnest engagement in behaviors that would be helpful to the maintenance of relationships (King \& Bond, 1985).

This paradoxical concept about relationship change implies that deliberate intervention is needed to manage changes that occur, whether intentional or natural change. It seems that being vigilant and realistic about the relationship process can be a source of motivation for individuals to do something about it, rather than feeling hopeless about the changing future of the relationship while doing nothing about it. Taken together, the findings suggest that changes are inevitable and yet this belief motivates individuals to introduce changes to the relationship in order to prevent the relationship from deteriorating. In other words, they actively engage in efforts to maintain the relationship before it takes the downward path. Hence, it seems that the three dimensions "Agent of Change", "Inevitable Change" and "Managing Change" essentially work hand-in-hand leading to an adaptive perspective and proactive attitude towards intimate relationships.

\subsection{Limitations}

The present study has several limitations that require careful consideration in interpretation and generalization of the results. Firstly, relationship is essentially a dyadic activity. Though the present study offered some insights to the dyadic-level representations of relationships in RBC, it is still an individual's cognitive representation. In intimate relationships, it is likely for a partner's beliefs to influence the other partner's psychological outcomes, especially in Chinese culture that promotes high interdependence in close relationships. For instance, a partner's belief about family resilience affects the subjective wellbeing of the other partner within the dyad (Neo, Chang, \& Fung, 2016). Hence, future research on the within or intra-dyad dynamic would be important to the under- 
standing of how relationship cognitions affect behaviors. Furthermore, as this is a within-culture study, hence no direct comparison has been made on change beliefs between the local participants and participants in other cultural context. Future cross-cultural studies, especially in comparison to individualistic cultures, would be beneficial for further exploring of young adults' relationship beliefs about change.

Furthermore, the utilization of different beliefs might be dependent on the nature and quality of the romantic relationship involved. Holmes (2002) suggested that the current state and health of the relationship might decide whether individuals would harness their uncertainty beliefs and anxiety in relationships and utilize it as form of motivation to care for the relationship. Different outcomes might arise from relationships that are generally satisfying and relationships that are already in a rocky or fragile state. Holmes contended that relationships that have had breached trust might not demonstrate improvement despite perceiving uncertainty and anxiety. Further studies would benefit from understanding the status of the relationship and relationship health before assessing their attachment tendencies and change beliefs.

\section{Conclusion}

The present study has presented a new construct of RBC based on the conceptualization of change in romantic relationships reported by young Singaporean Chinese. Relationships change is believed to involve proactively induced change and passive or natural change. In addition, change is accompanied by the perception that interventional effort is needed to manage the inevitable change in close relationships. This seemingly paradoxical belief of introducing change to curb potential change might have its roots in traditional Chinese culture that remains to influence the young adults in Singapore.

\section{References}

Bejanyan, K., Marshall, T. C., \& Ferenczi, N. (2015). Associations of Collectivism with Relationship Commitment, Passion, and Mate Preferences: Opposing Roles of Parental Influence and Family Allocentrism. PLoS ONE, 10, e0117374. https://doi.org/10.1371/journal.pone.0117374

Berscheid, E. (2010). Love in the Fourth Dimension. Annual Review of Psychology, 61, 1-25. https://doi.org/10.1146/annurev.psych.093008.100318

Byrne, B. M. (2010). Structural Equation Modeling with AMOS: Basic Concepts, Applications, and Programming (2nd ed.). New York, NY: Routledge.

Canevello, A., \& Crocker, J. (2011). Changing Relationship Growth Belief: Intrapersonal and Interpersonal Consequences of Compassionate Goals. Personal Relationships, 18, 370-391. https://doi.org/10.1111/j.1475-6811.2010.01296.x

Chan, D. K. S., Ng, T. T. T., \& Hui, C. M. (2010). Interpersonal Relationships in Rapidly Changing Chinese Societies. In M. H. Bond (Ed.), The Oxford Handbook of Chinese Psychology (pp. 515-532). New York, NY: Oxford University Press. https://doi.org/10.1093/oxfordhb/9780199541850.013.0031

Chang, S. C., \& Chan, C. N. (2007). Perceptions of Commitment Change during Mate Se- 
lection: The Case of Taiwanese Newlyweds. Journal of Social and Personal Relationships, 24, 55-68. https://doi.org/10.1177/0265407507072583

Chang, W. C., \& Sivam, R. W. (2004). Constant Vigilance: Heritage Values and Defensive Pessimism in Coping with Severe Acute Respiratory Syndrome in Singapore. Asian Journal of Social Psychology, 7, 35-53.

Chang, W. C., Toh, Y., Fan, Q., \& Chan, A. (2015). Mind over Body: Positive Attitude and Flexible Control Beliefs on Positive Aging. Biophilia, 3, 253-261.

https://doi.org/10.14813/ibra.2015.254

Chen, F., Curran, P. J., Bollen, K. A., Kirby, J., \& Paxton, P. (2008). An Empirical Evaluation of the Use of Fixed Cut-Off Points in RMSEA Test Statistic in Structural Equation Models. Sociological Methods and Research, 36, 462-494.

https://doi.org/10.1177/0049124108314720

Choi, I., Nisbett, R. E., \& Norenzayan, A. (1999). Causal Attribution across Cultures: Variation and Universality. American Psychological Bulletin, 125, 47-63. https://doi.org/10.1037/0033-2909.125.1.47

Collins, N. L., Guichard, A. C., Ford, M. B., \& Feeney, B. C. (2004). Working Models of Attachment: New Developments and Emerging Themes. In W. S. Rholes, \& J. A. Simpson (Eds.), Adult Attachment: Theory, Research, and Clinical Implications (pp. 196-239). New York, NY: The Guilford Press.

Dweck, C. S., Chiu. C. Y., \& Hong, Y. Y. (1995). Implicit Theories and Their Role in Judgments and Reactions: A World from Two Perspectives. Psychological Inquiry, 6, 267-285. https://doi.org/10.1207/s15327965pli0604_1

Eidelson, R. J., \& Epstein, N. (1982). Cognition and Relationship Maladjustment: Development of a Measure of Dysfunctional Relationship Beliefs. Journal of Consulting and Clinical Psychology, 50, 715-720.

https://doi.org/10.1037/0022-006X.50.5.715

Fletcher, G .J. O., Overall, N. C., \& Friesen, M. D. (2006). Social Cognition in Intimate Relationships. In A. L. Vangelisti, \& D. Perlman (Eds.), The Cambridge Handbook of Personal Relationships (pp. 353-368). New York, NY: Cambridge University Press. https://doi.org/10.1017/CBO9780511606632.020

Fraley, C. R., Waller, N. G., \& Brennan, K. A. (2000). An Item Response Theory Analysis of Self-Report Measures of Adult Attachment. Journal of Personality and Social Psychology, 78, 350-365. https://doi.org/10.1037//0022-3514.78.2.350

Gaines, S. O., Buriel, R., Liu, J. H., \& Rios, D. I. (1997). Culture, Ethnicity, and Personal Relationship Process. New York, NY: Routledge.

Goodwin, R., \& Tang, C. S. K. (1996). Chinese Personal Relationships. In M. H. Bond (Ed), The Handbook of Chinese Psychology (pp. 295-308). Hong Kong: Oxford University Press.

Hatfield, E., \& Rapson, R. L. (2010) Culture, Attachment Style, and Romantic Relationships. In P. Erdman, \& K-M. Ng (Eds.), Attachment: Expanding the Cultural Connections (pp. 227-242). London: Taylor Francis.

Hendrick, S. S. (1988). A Generic Measure of Relationship Satisfaction. Journal of Marriage and Family, 50, 93-98. http://www.jstor.org/stable/352430 https://doi.org/10.2307/352430

Hendrick, S. S., Dicke, A., \& Hendrick, C. (1998). The Relationship Assessment Scale. Journal of Social and Personal Relationships, 15, 137-142. https://doi.org/10.1177/0265407598151009

Hinde, R. A. (1997). Relationships: A Dialectical Perspective. Cambridge: Psychology Press. 
Holmes, J. G. (2002). Interpersonal Expectations as the Building Blocks of Social Cognition: An Interdependence Theory Perspective. Personal Relationships, 9, 1-26. https://doi.org/10.1111/1475-6811.00001

Hu, L. T., \& Bentler, P. M. (1999). Cut-Off Criteria for Fit Indexes in Covariance Structure Analysis: Conventional Criteria versus New Alternatives. Structural Equation Modeling: A Multidisciplinary Journal, 6, 1-55. https://doi.org/10.1080/10705519909540118

James, S., Hunsley, J., \& Hemsworth, D. (2002) Factor Structure of the Relationship Belief Inventory. Cognitive Therapy and Research, 26, 729-744. https://doi.org/10.1023/A:1021285332295

Ji, L. J., Lee, A., \& Guo, T. (2010). The Thinking Styles of Chinese People. In M. H. Bond (Ed.), The Oxford Handbook of Chinese Psychology (pp. 155-167). New York, NY: Oxford University Press. https://doi.org/10.1093/oxfordhb/9780199541850.013.0012

Ji, L. J., Nisbett, R. E., \& Su, Y. (2001). Culture, Change, and Protection. Psychological Science, 12, 450-456. https://doi.org/10.1111/1467-9280.00384

Kagitcibasi, C. (2007). Family, Self, and Human Development across Cultures: Theory and Applications (2nd ed.). Mahwah, NJ: Routledge Academic.

King, A. Y. C., \& Bond, M. H. (1985). The Confucian Paradigm of Man: A Sociological View. In W. Tseng, \& D. Y. H. Wu (Eds.), Chinese Culture and Mental Health (pp. 29-46). Orlando, FL: Academic Press.

Knee, C. R. (1998). Implicit Theories of Relationships: Assessment and Prediction of Romantic Relationship Initiation, Coping, and Longevity. Journal of Personality and Social Psychology, 74, 360-370. https://doi.org/10.1037/0022-3514.74.2.360

Knee, C. R., Patrick, H., \& Lonsbary, C. (2003). Implicit Theories of Relationships: Orientations toward Evaluation and Cultivation. Personality and Social Psychology Review, 7, 41-55. https://doi.org/10.1207/S15327957PSPR0701_3

Knee, C. R., \& Petty, K. N. (2013). Implicit Theories of Relationships: Destiny and Growth Beliefs. In J. A. Simpson, \& L. Campbell (Eds.), The Oxford Handbook of Close Relationships (pp. 183-198). New York, NY: Oxford.

Leung, K. (1996). The Role of Beliefs in Chinese Culture. In M. H. Bond (Ed), The Handbook of Chinese Psychology (pp. 247-262). Hong Kong: Oxford University Press.

Liu, W. Y. (2012). Conflict Management Styles in Romantic Relationships between Chinese and American Students: Exploring the Role of Cultural Individualism-Collectivism and Self-Construal (Paper 329). Open Access Theses.

http://scholarlyrepository.miami.edu/cgi/viewcontent.cgi?article $=1337 \&$ context=oa_th eses

MacCallum, R. C., Browne, M. W., Sugawara, H. M. (1996). Power Analysis and Determination of Sample-Size for Covariance Structure Modeling. Psychological Methods, 1, 130-149. https://doi.org/10.1037/1082-989X.1.2.130

Moore, S. M., \& Leung, C. (2001). Romantic Beliefs, Styles, and Relationships among Young People from Chinese, Southern European, and Anglo-Australian Backgrounds. Asian Journal of Social Psychology, 4, 53-68.

Schaerfl, C. A. (2009). Idiocentric and Allocentric Worldview Determinants of the Coparenting Alliance (Unpublished Doctoral Thesis). Fielding Graduate University. http://search.proquest.com/docview/305169520

Neo, A. H. C., Chang, W., \& Fung, D. (2016) An Actor-Partner Interdependence Model on Family Resilience and Subjective Wellbeing. Psychology, 7, 889-900.

https://doi.org/10.4236/psych.2016.76091 
Nisbett, R. E. (2003). The Geography of Thought: How Asians and Westerners Think Differently and Why. New York, NY: Oxford University Press.

Pietromonaco, P. R., \& Barrett, L. F. (2000). The Internal Working Models Concept: What Do We Really Know about the Self in Relation to Others? Review of General Psychology, 4, 155-175.

Rogge, R. D., \& Bradbury, T. N. (2002). Developing a Multifaceted View of Change in Relationships. In A. L. Vangelisti, H. T. Reis, \& M. A. Fitzpatrick (Eds.), Stability and Change in Relationships (pp. 228-253). Cambridge: Cambridge University Press. https://doi.org/10.1017/CBO9780511499876.013

Triandis, H. C. (1989). The Self and Social Behaviour in Differing Cultural Contexts. Psychological Review, 96, 269-289. https://doi.org/10.1037/0033-295X.96.3.506

Vangelisti, A. L., Reis, H. T., \& Fitzpatrick, M. A. (2002). Stability and Change in Relationships. Cambridge: Cambridge University Press. https://doi.org/10.1017/CBO9780511499876

Liang (1912). Like a Boat Sailing against the Current, We Must Forge Ahead or Be Swept Downstream. http://www.ccln.gov.cn/gbgx/myjj/178628.shtml

\section{Appendix: Relationship Beliefs about Change}

\begin{tabular}{|c|c|}
\hline 1. & Relationships change when I change. \\
\hline 2. & Relationships change when environment or situations change. \\
\hline 3. & Relationships change as growth (process of growth). \\
\hline 4. & Relationships change when I'm at different phases of my life. \\
\hline 5. & Relationships change when my partner changes. \\
\hline 6. & Relationships change as people are unpredictable. \\
\hline 7. & Relationships change as change is the only constant in life. \\
\hline 8. & Relationships change as relationship has different stages. \\
\hline 9. & Relationships change as a mean to compromise and work things out. \\
\hline 10. & Relationships change to "preserve" the relationship from going downhill. \\
\hline 11. & Without progress, things will deteriorate eventually, so as relationships. \\
\hline 12. & The longer the relationship, the more likely it will change. \\
\hline 13. & Relationships change because people like to experience the highs and lows in relationship. \\
\hline 14. & Relationships change when my perception of my partner changes. \\
\hline
\end{tabular}


Submit or recommend next manuscript to SCIRP and we will provide best service for you:

Accepting pre-submission inquiries through Email, Facebook, LinkedIn, Twitter, etc. A wide selection of journals (inclusive of 9 subjects, more than 200 journals)

Providing 24-hour high-quality service

User-friendly online submission system

Fair and swift peer-review system

Efficient typesetting and proofreading procedure

Display of the result of downloads and visits, as well as the number of cited articles Maximum dissemination of your research work

Submit your manuscript at: http://papersubmission.scirp.org/

Or contact psych@scirp.org 